\title{
The Role of Neuroimaging and Electroencephalogram in Diagnosis of Alzheimer Disease
}

\author{
N. N. Kulkarni \\ Lecturer, E and TC Engg. \\ Sou. Venutai Chavan \\ Polytechnic, Pune -41
}

\author{
P. P. Rathod \\ Lecturer, E and TC Engg. \\ Sou. Venutai Chavan \\ Polytechnic, Pune - 41
}

\author{
V. V. Nanavare \\ Lecturer, E and TC Engg. \\ Sou. Venutai Chavan \\ Polytechnic, Pune - 41
}

\begin{abstract}
Alzheimer Disease is the neurodegenerative disease, which is the common form of dementia. It is the most expensive disease in the modern society characterized by cognitive, intellectual as well as behavioral disturbance. Therefore, early diagnosis of the disease is essential. The disease progressively can lead to the total dependency at the severe stage. Different techniques for early diagnosis of Alzheimer disease including neuroimaging techniques and non-neuroimaging techniques can be effectively used. Computer Aided Diagnosis tools plays a vital role in computer based diagnosis. Besides this, non-neuroimaging techniques such as Biomarkers, Electroencephalography can be used as standardized tools for diagnosis of Alzheimer Disease. This paper discusses the important aspects of the above techniques in early diagnosis of Alzheimer disease. In this paper, comparison of the different diagnosis tools for diagnosis of Alzheimer disease is presented. The paper discusses the role of Electroencephalography in diagnosis of Alzheimer disease and how Electroencephalography plays an important role in diagnosis compared to other methods. Today, the role of EEG in diagnostic and clinical research of Alzheimer disease has become more useful. Thus, EEG can be as the tool for the early diagnosis of Alzheimer disease.
\end{abstract}

\section{Keywords}

Alzheimer Disease; Dementia; Electroencephalography; Magnetic Resonance Imaging; Positron Emission Tomography ;Single Photon Emission Computed Tomography.

\section{INTRODUCTION}

Alzheimer Disease (AD) is one of the Neuro-degenerative diseases which are found to be complex in the present scenario. It is the common form of dementia and by the time it affects the brains cells [1] [2]. Alzheimer Disease is a chronic Neuro-degenerative disorder that has ranked as third most expensive disease and sixth leading cause of death in United States. It is neurodegenerative disorder characterized by rapid impairment of memory and some other cognitive functions, which are mainly associated with the behavioral disturbances and finally leads to total dependency [3]. An important research is to identify the neuroanatomical basis of cognitive impairment in Alzheimer Disease (AD). The need of research is to understand the changes taking place related to the cognitive impairment and the progression of $\mathrm{AD}$ in the brain structure [4] - [7].

$\mathrm{AD}$ is common outcome of Dementia as the stage progresses. The beginning of the disease starts with the neuronal dysfunction and irreversible loss of neurons. Mild Cognitive Impairment (MCI) is the intermediate state between normal aging and severe $\mathrm{AD}$ in which density and distribution of tau- associated neurofibrillary and beta associated amyloid for a long time exist. MCI indicates the increased risk of progression of $\mathrm{AD}$. In same stage, memory loss and impairment is noticed. Language Difficulty, Paraphasia are some of the deficits seen in the moderate stage. The final stage of disease called - Severe $\mathrm{AD}$, all cognitive functions are severely damaged, motor functions such as chewing and swallowing are also found disturbed [8]. Basically, there are no proper symptoms for cause of AD. But, there exists some genetic differences along with the different hypothesis used for understanding the cause of Alzheimer Disease. Some common hypothesis includes the genetic cholinergic hypothesis, amyloid hypothesis, tau hypothesis etc. One important hypothesis regarding the cause of $\mathrm{AD}$ is due to age related myelin breakdown in the brain. Early diagnosis of disease in early stage is essential in both Mild AD and MCI stage as medication can be applied in an early stage. At final stage of disease, patient cannot be cured and hence it can lead to death. Thus, diagnosis in early stage is essential. On the other hand, early is diagnosis allows the patients family to take financial decisions related to disease and to plan the future needs involving the care of patient. Presently, it is estimated that there are 44.4 millions of people suffering from dementia in the world. It is also estimated that this number will increase till 75.6 million in 2030 , and 135.5 million in 2050. It was also observed that $61 \%$ of the people suffering from dementia are from the developed countries. The fastest growth in the elderly population is taking place in China, India, and their south Asian and western Pacific neighbors [9] [8].

In further part of the paper, various neuroimaging methods such as MRI (Magnetic Resonance Imaging), SPECT (Single Photon Emission Computerized Tomography), PET (Positron Emission Tomography) and other non-neuroimaging methods like EEG, biomarkers etc. are discussed to diagnose the disease in early stage. The paper also discusses the methodology for EEG Based diagnosis of Alzheimer Disease. In result section, slowing effect and reduction of Power in higher frequency bands and increase in Power of lower frequency bands observed in the EEG signal of Alzheimer Disease patients is also discussed.

\section{NEUROIMAGING AND RADIOLOGY IN DIAGNOSIS OF ALZHEIMER DISEASE}

Numerous clinical methods are extensively used for the diagnosis of Alzheimer disease such as neuroimaging techniques, physiological markers, and genetic analysis. Neuroimaging is one of the well-accepted methods for definitive diagnosis of dementia. As it known that there is no cure for Alzheimer disease but early diagnosis of $\mathrm{AD}$ is an 
effective treatment. For diagnosis, information extracted from $3 \mathrm{D}$ brain images is the principal behind the development of automatic tool [10]. Neuroimaging techniques increase the confidence of diagnosis. Along with this, Computer Aided Diagnosis (CAD) system uses the discriminative value of brain images produced by different neuroimaging techniques such as MRI, PET, and SPECT to identify the patient suffering from Alzheimer Disease, Mild Cognitive impairment (MCI), Dementia or whether normal.

Imaging has a key role in medical diagnosis, education and noninvasive therapeutics. The new scientific and technological advances boosts the complex issues of diseases such as Alzheimer, epilepsy etc. The interaction of computers and technology made imaging a necessity due to possibility in diagnosis of disease along with providing the treatment. Medical imaging is then useful whenever there is a need to diagnose the early condition of disease, planning of a surgery, or for radiation therapy. Medical Imaging is regarded as a pillar in clinical as well as medical applications. Computer tomography (CT), or Magnetic Resonance Imaging (MRI), called Conventional structural neuroimaging plays a supportive role in diagnosis of memory disorder. Along with CT and MRI, emission tomography techniques also play a major role in clinical diagnosis of diseases. The emission based images termed as functional images represents the physiological functions with anatomical structure images providing information about physiological phenomenon. As previously stated CAD is a general tool used for a variety of application such as to diagnose the disease in medical applications. CAD helps the physicians, researchers in diagnosing the disease in less time by identifying he patterns, making fewer efforts [11].

Let us discuss some important and widely used neuroimaging techniques used in early diagnosis of $\mathrm{AD}$ as well as Dementia.

\subsection{MRI (Magnetic Resonance Imaging)}

Neuroimaging with MRI plays a vital role in diagnosing of dementia as well as Alzheimer disease. MRI is also a superior tool for brain imaging due to absence of ionizing radiations, increased image flexibility and better tissue contrast. Magnetic Resonance is also stated a reliable supportive tool for the diagnosis It reveals cortical atrophy, sulcal and ventricular dilatations, hippocampal volume and some related change in parenchymal intensity [12] [13]. MRI imaging enables the accurate differentiation between individuals with Mild cognitive impairment (MCI), and probable Alzheimer disease.

Some other types of MR techniques include MR perfusion imaging, functional MR imaging, MR spectroscopy and diffusion weighted MR imaging. MR perfusion imaging is a method that uses the rapid $\mathrm{T} 2 *$-weighted imaging of brain. This method enables measurement of several hemodynamic parameters. Similarly, in functional MR imaging regional brain activity is measured on local changes in deoxyhaemoglobin concentrations. On other hand, MR Spectroscopy is an application of MR allowing non-invasive assessment of a no of number of local metabolite label in brain tissue. Diffusion-weighted MRI imaging is another technique which is sensitive to the microscopic motion of water molecule in brain tissue. The all sub types of MRI imaging plays a significant role in study of patient with Alzheimer disease [14] [15].

\subsection{PET (Positron Emission Tomography)}

PET is also one of the clinical diagnosis method used in differentiating Alzheimer disease, dementia and normal patient. Positron emission tomography is nuclear medicine imaging technique in which operating principle relies on detection of pair of gamma rays which are emitted by a positron emitting radionuclide called tracer. PET is introduced into the body on a triologically active module. In PET neuroimaging technique, tomography performed on brain shows reduction of metabolism in certain areas of brain associated with Alzheimer disease patients [16]. PET is also used to study the brain's metabolic uptake of fluorine 18 (18F)-labeled fluorodeoxyglucose (FDG) and blood flow in patients with dementia. Along with this diagnostic accuracy of FDG-PET is defined in terms of sensitivity (true positive) and specificity (true negative). Clinical assessment serves as a reference standard for FDG-PET evaluations for diagnosing of Dementia and Alzheimer disease [17].

Thus, PET is a neuroimaging technique which provides information about physiological and biochemical process. It is also referred as a useful biomarker for diagnosis of dementia and Alzheimer disease. On the same platform, it helps in determining whether the patient is normal or suffers from cognitive dysfunction [18]. Positron Emission tomography plays therefore a key role in differentiation of neurological disease.

\subsection{SPECT (Single Photon Emission Computed Tomography)}

SPECT is a molecular imaging technique which provides information as same that of PET. SPECT has a lower resolution then PET. SPECT is based on a brain uptake of a technetium 99mbased lipid soluble radionuclide such as ethyl cysteinate dimer or hexamethylpropylene which is locally available for evaluating brain perfusion with the help of rotating gamma camera. It is widely used technique for evaluation of brain perfusion with a rotating gamma camera. SPECT imaging provides additional diagnostic value tool for patients with dementia. It is classic noninvasive tool used for diagnosing of dementia and its later stages such MCI and Alzheimer disease. SPECT measures the regional blood flow, whereas fluorodeoxyglucose positron emission tomography (FDG-PET) examines glucose metabolism in brain [10].

Thus, the above of the neuroimaging methods proves to be useful in diagnosing the Alzheimer Disease and its subsequent phases.

\section{NON-NEUROIMAGING}

\section{TECHNIQUES FOR DIAGNOSIS OF ALZHEIMER DISEASE}

As seen in previous section neuroimaging techniques plays an important role in diagnosis of neurodegenerative diseases such as dementia, Alzheimer etc. Neuroimaging techniques are well-accepted methods for diagnosis of Alzheimer disease. But, it has certain disadvantages. The major problem of neuroimaging techniques includes the radiation risks. Apart from this, Neuroimaging techniques such as MRI, SPECT, PET, fMRI etc. are found to be much expensive, time consuming and inconvenient. In such cases, nonneuroimaging techniques plays an vital role in diagnosis of diseases.. The advantages of non-neuroimaging techniques includes that they are inexpensive, repeatable, and can be easily used at home through wireless networks. In future nonneuroimaging methods such as Electroencephalography can also be made portable [19]. 
Non-neuroimaging techniques such as Biomarkers, Electroencephalography (EEG) are widely accepted methods for diagnosis of Alzheimer disease. Cerebrospinal Fluid (CSF) and Plasma Biomarkers, genetic biomarkers are some of the biomarkers used in diagnosis of Dementia and Alzheimer disease [10]. The core diagnostic cerebrospinal fluid markers for diagnosis of Alzheimer disease and Dementia include A $\beta 42$, T-tau, and P-tau. According to Blennow et al. [20], the low level concentration of $A \beta 42$ and high level of $P$ or $T$ - tau identify $\mathrm{AD}$ with sensitivity and specificity of $\mathrm{AD}$ with sensitivity of $80 \%$. Other biomarkers such as Genetic Biomarkers also play a major in diagnosis of AD. Amyloid Precursor protein (APP), Presenilin-1(PSEN1), and Presenilin-2 (PSEN2) are well accepted susceptibility genes for early diagnosis of Alzheimer disease. Above biomarker and imaging techniques have limitations to their use although they have much development. The reason is due to the collection of CSF is difficult and uncommon for diagnosis of dementia and Alzheimer disease. It is also an invasive method. Electroencephalography (EEG) is one of the tools which can be used for early diagnosis of Alzheimer disease. EEG is noninvasive, repeatable and can be easily evaluated. It is a personalized medical tool. EEG can be used for direct correlation of brain function, which can be used for monitoring brain activity. Nonlinear analysis of EEG data shows the unique features to reveal the diagnosis of neurological diseases such as Alzheimer, Epilepsy and Parkinson's [21] [22].

As compared to the other Neuro-imaging methods, EEG has high temporal resolution and as a consequence several abnormalities are found in the EEG of the patients. Let us now discuss use of EEG for diagnosis of Dementia and Alzheimer disease in brief.

\subsection{EEG based diagnosis of Alzheimer disease}

Electroencephalography (EEG) is a non-invasive tool for diagnosis of neurological disease. The advantages of EEG are discussed in above section. Certain abnormalities are found in EEG signals of patients suffering from Alzheimer disease [23] [24] [25] [26] [27]. The various abnormalities found in patients suffering from Alzheimer disease includes (i) Slowing of EEG signals, (ii) Reduced Complexity of EEG signals and (iii) Perturbation in EEG synchrony [28] [29] [30] [31] [32] [33] . It is important to focus on these three abnormalities for early diagnosis of Alzheimer disease.

Let us now discuss the above abnormalities in brief.

\section{A. Slowing of EEG Signals}

It is important phenomenon found in EEG signal. The slowing of EEG is mainly associated due to MCI, and $\mathrm{AD}$. It is also associated with increase in the power of low frequency (delta and theta band, $0.5-8 \mathrm{~Hz}$ ), and a decrease of power in higher frequency (alpha-beta, 8-30 $\mathrm{Hz}$ ). From fig. 1 we can observe that transient oscillation in EEG of Alzheimer patients occur at low frequencies compared to healthy control subjects. Thus, the transients exhibit the slowing of signals.

\section{B. Reduced Complexity of EEG Signals}

Several techniques are available for reducing the complexity in EEG signals for accurate diagnosis of Alzheimer disease. Different methods including information theory [35] [36], Tsallis entropy [36], approximate entropy [37], multiscale entropy [39], sample entropy [38] and auto - mutual information [40] and Lempel-Ziv complexity [41] [42] are used to quantify EEG complexity.

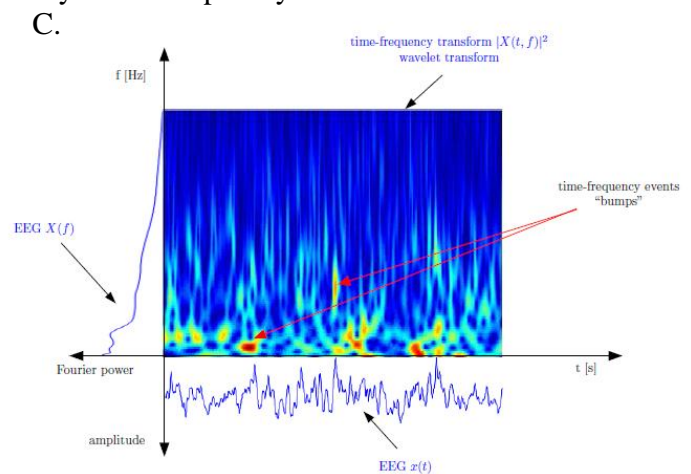

Fig 1: Slowing of EEG Signals [34]

\section{Perturbation in EEG Synchrony}

The various methods of synchrony measures are used in physical sciences, signal processing, and study of neurobiological sciences. The results have cleared that EEG synchrony decreases in patients with $\mathrm{MCI} /$ Alzheimer disease. Synchrony measures obtained from EEG signals are tremendously affected. The synchrony measures used for early diagnosis of Alzheimer disease from EEG signals includes Pearson Correlation Co-efficient, Magnitude and Phase Coherence, Granger Causality, Phase Synchrony etc.

\subsection{Method for diagnosis of Alzheimer disease}

The diagnosis of Alzheimer disease using EEG signals is followed by suitable methodology. Initially, EEG signal from patient is acquired through the EEG electrode. Basically, 10 20 electrode placement system is used for acquiring of EEG signal. Now-days, a special electrode cap is available in market, which is normally used for electrode placement on patents head. Once the signal is acquired, a pre-processing of EEG is done. This is done in order to remove certain artifacts available in signal. In preprocessing, the signal is also digitalized. Signal is digitalized in order to interface with computer. In order to enhance the EEG signal, Blind source separation, Independent component analysis, Principal Component analyses are used. Once the signal is processed and interfaced with computer, analysis of signal is done by using standard tools such as MATLAB. By using suitable feature extraction methods, various algorithms are used for differentiating the signal. Various techniques like Fast Fourier transform (FFT), Bump modeling are some of the methods. The features extracted are given as the input for classification purpose. Various classifiers are available for distinguishing between normal patient and patient suffering from Alzheimer disease. Linear Discriminant Analysis, Support vector machine are some of the classifiers that can be used for diagnosis [19].

The fig. 2 shows the methodology used for early diagnosis of $\mathrm{AD}$ using EEG signals.

\subsection{Subjects}

Data used in the study was obtained from Smt. Kashibai Navale General Hospital and Research Centre consisting of both Alzheimer patients and control patients. Patients were selected from consecutive, community residing elderly persons 55-80 years of age with the report of decline in 
cognitive as well as behavioral functioning. Diagnosis of the patients was made by experienced neurologists based on Indian version of Mini Mental State Examination (MMSE) and Clinical Dementia Rating (CDR). Resting awake multi channel EEG recordings were obtained from 25 participants separated into 2 groups. The first group was composed of 5 subjects; 3 males and 2 females (mean age: 60 years) giving indication of functional cognitive and behavioral decline. The second group consists of 20 participants of normal subjects consisting of 15 males and 5 females (mean age: 60.5 years), giving no indication of functional cognitive decline. In addition to the $\mathrm{AD}$ cohorts, an additional criterion was the presence of functional, behavioral and cognitive decline over the previous 6 months. Patients belonging to the abnormal group were also checked for diabetes, kidney disease, thyroid disease lung and liver disease or vitamin B12 deficiency, as these can also cause cognitive decline. The EEG recordings and the study was approved from Ethical committee of the hospital and the participants.

\subsection{Data Acquisition and Preprocessing}

EEGs were recorded from RMS (Recorders and Medicare systems Private Limited) EEG machine with 12 bits resolutions and sampling rate of $1024 \mathrm{~Hz}$. Impedance was maintained below 10Mohms and the electrodes (Referential Montages) were placed according to the International 10-20 systems. Biauricular referential electrodes were also attached as recommended by American EEG Society. The Power grid interference was eliminated by low pass filtering. As there is evidence of an interhemisperic disconnection in $\mathrm{AD}$ and dementia, a virtual hemisphere bipolar montage is also taken into consideration. The obtained signals are also termed as 'Bipolar signals'. The Bipolar signals recorded in the study and taken into the consideration are Fp1-Fp2, F3-F4, F7-F8, C3-C4, T3-T4, P3-P4, T1-T6, O1-O2,. During EEG examination and recordings, patients were awake and relaxed with eyes closed. The artifacts of EEG signals such as muscle activity and eye blinking were removed manually.

\section{RESULTS}

Dauwels et al. [34] have already said that EEG signals of Alzheimer Disease patients exhibits slowing effect. In our experiments, we have computed the power of the EEG signal in different EEG Sub bands such as Delta $(0.1-4 \mathrm{~Hz})$, Theta
$(4-8 \mathrm{~Hz})$, Alpha $(8-12 \mathrm{~Hz})$, Beta $(13-30 \mathrm{~Hz})$, and Gamma $(30-100 \mathrm{~Hz})$ bands. Firstly, we have obtained the raw EEG signal from the RMS machine in .eeg format. The EEG data is loaded into the MATLAB. For the detail analysis of the EEG signal, we have classified the same EEG signal into the different EEG sub bands by use of Wavelet Decomposition function. For Wavelet Decomposition in our experiment, we have used the Daubechies Wavelet ("db8") with level of Decomposition as 8. The Wavelet Decomposition technique is used in our experiment for classifying the EEG into different bands. The brief procedure of Wavelet Decomposition is given below. In general wavelet analysis, we mainly are concerned about the approximations and details coefficients of the signals. The approximations are the high-scale, lowfrequency components of the signal while the details are the low-scale, high-frequency components of the signal. Basically, the original signal is passed through the above two complementary filters and emerges as two signals. These both $\mathrm{A}$ and D signals and their values are important. But, still there exists the method to decompose the signal using wavelets. The computation of the co-efficient play an important role since we need to keep only one point out of two in each of the two n-length samples to get the complete information. This is the idea of downsampling. These produce two sequences called $\mathrm{cA}$ and $\mathrm{cD}$.

The downsampling process produces the wavelet coefficients. In our experiment, we are interested in Multi-level decomposition level since we want to break the signal in the lower resolution components. The process of multi level decomposition is iterative and requires successive approximation. This is called Multi Level Decomposition Tree. After decomposing by the same process, we have classified the raw EEG signal in the different sub bands. The following figure shows the output of the classification of the EEG signals into the various bands.

The power of the EEG signal in the various sub bands can also be calculated. Similarly, scalogram of the raw EEG signals using the wavelet transform is also computed using wavelet transform. Fig 3 show the slowing effect of the EEG signal observed in EEG signal of Alzheimer's disease patient. The red dots denote the "bumps" observed in the scalogram which exhibits the phenomenon of the slowing effect.

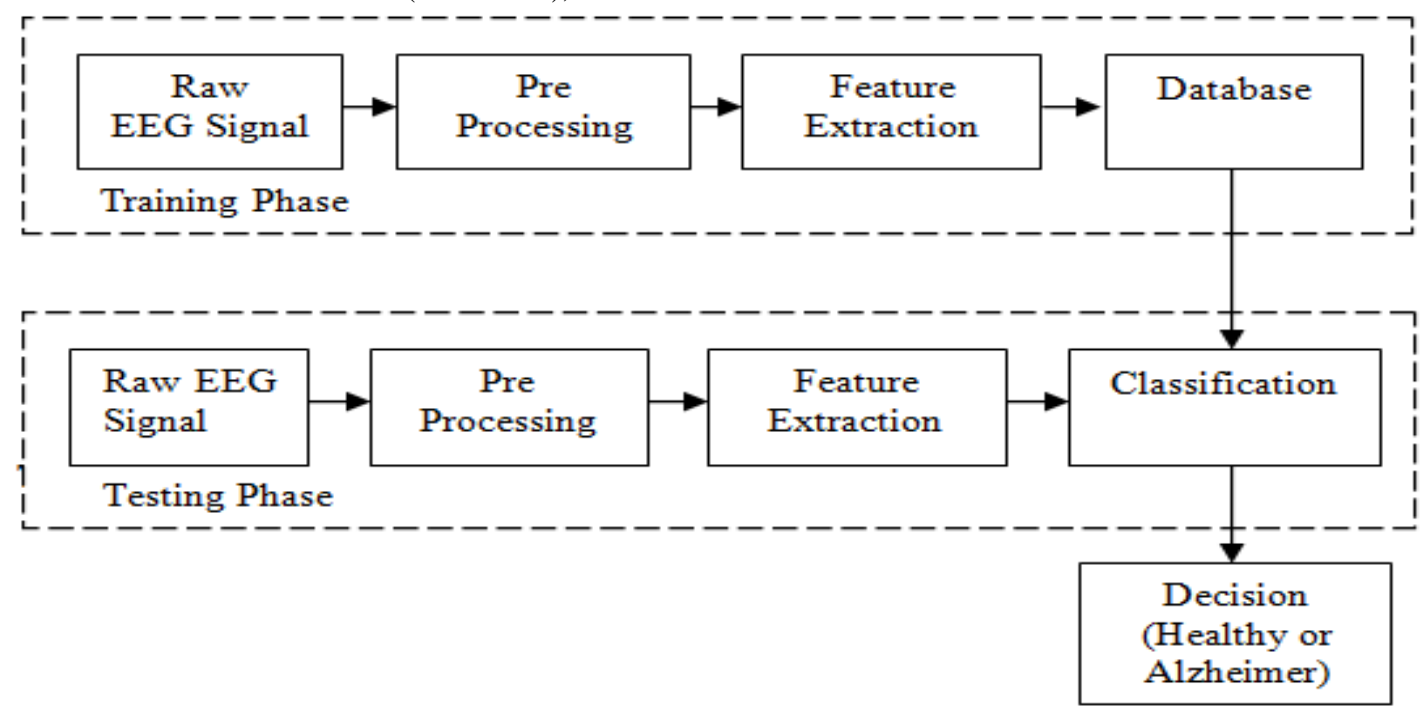

Fig 2: Methodology used for diagnosis of Alzheimer disease using EEG Signals 


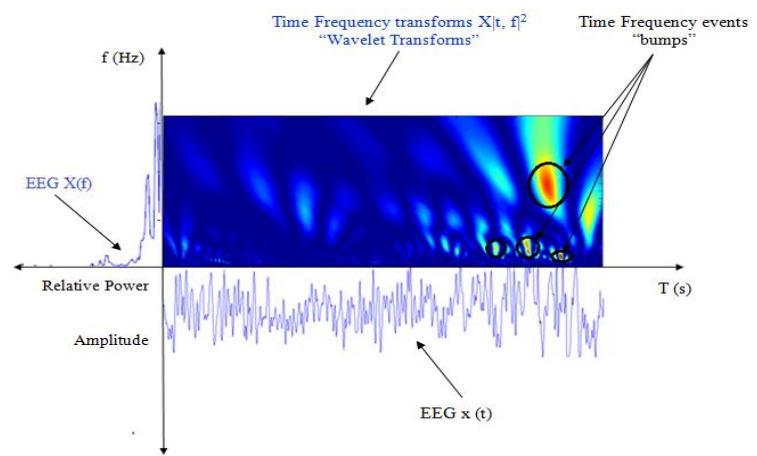

Fig 3: Slowing of EEG Signals in time, frequency and time-frequency domain

\section{DISCUSSIONS}

From the view of above information, we can get up to the point that different diagnosis techniques are available for diagnosis of Alzheimer disease as well as dementia. Neuroimaging as well as non - neuroimaging techniques has great significance in diagnosis of neurodegenerative diseases. Apart from this, Computer Aided diagnosis has also a tremendous scope for development in automated diagnosis of Alzheimer disease. From the survey, it can be concluded that neuroimaging imaging techniques prove to be beneficial for early diagnosis of Alzheimer disease. But, it has several negative impacts. Firstly, there is a problem of imposing radiation risks on patients. Others such disadvantages include they are expensive, inconvenient and time consumable. Apart from these, Biomarkers [18] and EEG [25] are also the tools used for diagnosis of disease. Various diagnosis tools are discussed in the previous sections of this article. Different neuroimaging techniques such as MRI, SPECT and PET are discussed. The advantages of using MRI includes its high flexibility, high temporal and spatial resolution absence of ionizing radiations but includes certain disadvantages such as its high cost, time consumable, delay in data acquisition process and large structure (hence requires more space) [19] [43]. Similarly, SPECT is also an advanced tool which is useful in diagnosing the neurological disorders such as Alzheimer disease as it measures the rate of blood flow in brain and FDG-PET is another advanced technique which measures the glucose metabolism in brain which is helpful in diagnosis of Dementia and Alzheimer disease [10]. Lastly, PET is another neuroimaging technique which is used in diagnosis of Alzheimer disease. The PET shows the reduction in metabolism in certain part of brain of people suffering from dementia as well as Alzheimer. Although the PET has an application in medical, clinical diagnosis of various disease such as Cancers, Neurological disorders but it has certain limitations which includes the high costs of PET machines. Similarly, high costs of cyclotrons needed to produce radionuclides for PET scanning purposes.

Today many medical researchers and doctors are although diagnosing the Alzheimer disease from Neuroimaging and clinical examinations; but in future EEG can be used as the diagnostic tool for automated diagnosis of dementia as well as Alzheimer disease. From the above discussions, it is concluded that EEG can be used as one of the important tool for diagnosis of Alzheimer disease. EEG is inexpensive tool as compared to other neuroimaging techniques and can be easily available. Diagnosis of Alzheimer disease using EEG signals has great impact on EEG signals. Slowing of EEG signals, Perturbations in Synchrony measures and reduced complexity are some of the abnormalities seen. Using the methodology shown in Fig. 2, method for early diagnosis of
Alzheimer disease using EEG signals can be implemented. From the above methodology, we have also computed the power obtained in various EEG Sub bands such as Delta, Theta, Alpha, Beta and Gamma. The Power in low frequency bands increases in $\mathrm{AD}$ infected patients whereas the power in the higher frequency bands decreases. Similarly, we have also observed the slowing phenomenon taking place in the EEG signal of $\mathrm{AD}$ patients. In future various different classification algorithms and different features can be used for increasing the diagnosis accuracy of Alzheimer disease [43].

Table. 1 shows the comparison of all the techniques used for Alzheimer disease with their use and advantages discussed in the paper.

Table 1: Comparison of various techniques for diagnosis of Alzheimer's disease

\begin{tabular}{|c|c|c|c|}
\hline Technique & Progression & Non - invasiveness & Cost \\
\hline PET & No & Yes & High \\
\hline MRI & No & Yes & High \\
\hline SPECT & Yes & No & High \\
\hline EEG & Yes & Yes & Low \\
\hline
\end{tabular}

\section{ACKNOWLEDGMENTS}

Authors gratefully acknowledge entire staff and non-teaching staff of Electronics and Telecommunication Engineering department for their kind cooperation in present research work. Author would also like to acknowledge principal of the institute for providing necessary support in present research work.

\section{REFERENCES}

[1] Mattson M., Pathways towards and away from Alzheimer's disease, Nature, Vol. 430, pp. 631-639. 2004.

[2] Meek P.D., McKeithan K., and Shumock G.T., Economics considerations of Alzheimer's disease, Pharmacotherapy, Vol 18, pp.68-73, March-April 1998.

[3] Jing Wan, Zhilin Zhang, Bhaskar D. Rao, Fellow, IEEE, Shiaofen Fang, Jingwen Yan, Andrew J. Saykin, Li Shen, Identifying the Neuroanatomical Basis of Cognitive Impairment in Alzheimer's Disease by Correlation and Nonlinearity-Aware Sparse Bayesian Learning, IEEE Transactions on Medical Imaging, Vol.33, No.7, pp.1475-1487, July 2007.

[4] S. Klöppel et al., Automatic classification of MR scans in Alzheimer's disease, Brain, Vol. 131, No. 3, pp. 681689, 2008.

[5] C. Plant et al., Automated detection of brain atrophy patterns based on MRI for the prediction of Alzheimer's disease, Neuroimage, Vol. 50, No. 1, pp. 162-174, 2010.

[6] M. Radanovic et al., White matter abnormalities associated with Alzheimer's disease and mild cognitive impairment: A critical review of MRI studies, Expert Rev. Neurotherapeut., Vol. 13, No. 5, pp.483-493, 2013.

[7] A. Chincarini et al., -Alzheimer's disease markers from structural MRI and FDG-PET brain images, Eur. Phys. J. Plus, Vol. 127, no. 11, pp.1-16, 2012. 
[8] Bianchetti A, Trabucch M., Clinical aspects of Alzheimer's disease. AgingClin Exp Res, Vol. 13, pp. 221-30, 2001.

[9] Béatrice Duthey, A Public Health Approach to Innovation, Update on 2004 Background Paper written by SaloniTanna, February 2013.

[10] Gorsev G.Yener, Erol Basar, Biomarkers in Alzheimer's disease with a special emphasis on event related oscillatory responsesll, Application of Brain Oscillations in Neuropsychiatric Diseases (supplements to Clinical Neurophysiology), Vol. 62, pp. 237-273, 2013.

[11] P. Padilla, M. López, J. M. Górriz, J. Ramírez, D. SalasGonzález, I. Álvarez, and The Alzheimer's disease Neuroimaging Initiative, NMF-SVM Based CAD Tool Applied to Functional Brain Images for the diagnosis of Alzheimer Disease, IEEE Transactions on Medical Imaging, Vol. 31, No. 2, pp. 207-216, February 2012.

[12] Knopman DS, Boeve BF, Petersen RC., Essentials of the proper diagnoses of mild cognitive impairment, dementia, and major subtypes of dementia, Mayo Clin Proceedings, Vol. 78, pp. 1290-308, 2003.

[13] Steffens DC, McQueen DR, Payne ME, et al., Change in hippocampal volume on magnetic resonance imaging and cognitive decline among older depressed and nondepressed subjects in the neurocognitive outcomes of depression in the elderly study, Am J Geriatr Psychiatry, Vol. 19, pp. 4-12,2 011.

[14] Hanyu H, Sakurai H, Iwamoto T, Takasaki M, Shindo H, Abe K., Diffusion weighted MR imaging of the hippocampus and temporal white matter in Alzheimer's disease., J Neurol Sci, Vol.156, pp.195-200,1998.

[15] Hanyu H, Shindo H, Kakizaki D, Abe K, Iwamoto T, Takasaki M., Increased water diffusion in cerebral white matter in Alzheimer's disease, Gerontology, Vol. 43, pp. 343-351, 1997.

[16] D. Silverman, PET in the Evaluation of Alzheimer's disease and Related Disorders. Springer, 2009.

[17] Shokouhi S, Classen D, Riddle WR, Imaging Brain Metabolism and Pathology in Alzheimer's disease with positron Emission Tomography, Journal of Alzheimer's Disease \& Parkinsonism, 2014.

[18] Marilyn Albert et al., The use of MRI \& PET for Clinical Diagnosis of Dementia and Investigation of Cognitive Impairment: A Consensus Reportl, Prepared by the Neuroimaging Work Group of the Alzheimer's Association.

[19] Nilesh Kulkarni, V. K. Bairagi, Diagnosis of Alzheimer Disease using EEG Signalsl, International Journal of Engineering Research \& Technology, Vol. 3,No. 4, 2014.

[20] Blennow, K., Hampel H., Weiner, M. and Zetterberg, H., Cerebrospinal fluid and plasma biomarkers in Alzheimer disease, Nat. Rev. Neurol., Vol. 6, pp.131$144,2010$.

[21] Beuter, C. Labric, and K. Vasilakos, Transient dynamics in motor control of patients with Parkinson's disease, Chaos, Vol. 1, pp. 279-286, 1991.

[22] H. L. Glass, Nonlinear dynamics of physiological function and control, Chaos, Vol. 1, pp. 247-250, 1991.
[23] Sink K.M., Holden K.F., and Yaffe K., Pharmacological treatment of neuropsychiatric symptoms of dementia: a review of the evidence. JAMA, Vol 293, Issue 5, pp. 596-608, 2005.

[24] Baker M., Akrofi K., Schiffer R., and Michael W. O' Boyle, EEG Patterns in Mild Cognitive Impairment (MCI) Patients., Open Neuroimage J., Vol. 2, pp. 52-55, August 2008.

[25] Besthorn C., Zerfass R., Geiger-Kabisch C., Sattel H., Daniel S., Schreiter-GasserU., and Frstl H., Discrimination of Alzheimer's disease and normal aging by EEG data., Electroencephalography and Clinical Neurophysiology, Vol. 103, No. 2, pp.241-248, August 1997.

[26] Van der Hiele K., Vein A.A., Reijntjes R.H., Westendorp R.G., Bollen E.L., van Buchem M.A., Van Dijk J.G., Middelkoop H.A., EEG correlates in the spectrum of cognitive decline., Clinical Neurophysiology, Vol. 118, No. 9, pp.1931-1939, September 2007.

[27] Czigler B., Csik'os D., Hidasi Z., Anna Ga’al Z., Csibri E., Kiss E., Salacz P., Moln'ar M., Quantitative EEG in early Alzheimer's disease patients power spectrum and complexity features. , Int. J. Psychophysiol. , Vol. 68, No. 1, pp. 75-80, April 2008.

[28] Gianotti L.R., K"unig G., Lehmann D., Faber P.L., Pascual-Marqui R.D., Kochi K., and Schreiter-Gasser U., Correlation between disease severity and brain electric LORETA tomography in Alzheimer's disease., Clin.Neurophysiol., Vol. 118, No. 1, pp. 186-96, January 2001

[29] Herrmann C.S. and Demiralp T., Human EEG gamma oscillations in neuropsychiatric disorders, Clin. Neurophysiology. Vol. 116, No. 12, pp. 2719-2733, December 2005.

[30] Latchoumane C.F.V., Vialatte F.B., Cichocki A., and Jeong J., Multiway analysis of Alzheimer's disease: classification based on space-frequency characteristics of EEG time series, Proc. World Congress on Engineering 2008, London, United Kingdom, 2-4 July, 2008.

[31] Latchoumane C.F.V, Vialatte F.B., Jeong J., and Cichocki A. EEG Classification of Mild and Severe Alzheimer's Disease using Parallel Factor Analysis Method. In Advances in Electrical Engineering and Computational Science, in press, 2009.

[32] Schreiter Gasser U., Rousson V., Hentschel F., Sattel H., Gasser T., Alzheimer disease versus mixed dementias: an EEG perspective., Clin Neurophysiology, Vol. 119, No. 10, pp. 2255-2759, October 2008.

[33] Moretti D.V., Fracassi C., Pievani M., Geroldi C., Binetti G., Zanetti O., Sosta K., Rossini P.M., and Frisoni G.B., Increase of theta/gamma ratio is associated with memory impairment. Clin.Neurophysiol., Vol. 120, No. 2, pp. 295-303, February 2009.

[34] Justin Dauwels, Francois Vialatte, and Andrzej Cichocki, Diagnosis of Alzheimer's disease from EEG Signals: Where Are We Standing?, Current Alzheimer Research, Vol. 7, No. 6, September 2010.

[35] Shannon C.E., A Mathematical Theory of Communication. Bell System Technical Journal, Vol. 27, pp. 379-423, 623-656, July-October 1948. 
[36] Cover T. M. and Thomas J. A., Elements of Information Theory. Wiley, New York, 1991.

[37] Pincus S.M., Approximate entropy as a measure of system complexity, Proc.Natl Acad Sci, USA, Vol 88, pp. 2297-2301, March 1991.

[38] Lake D.E., Richman J.S, Griffin M.P., and Moorman J.R., Sample entropy analysis of neonatal heart rate variability, Am J Physiol, Vol. 283, No. 3, pp. 789-797, September 2002.

[39] Costa M., Goldberger A.L., Peng C.-K., Multiscale entropy analysis of physiologic time series., Phys Rev Lett, Vol. 89, No. 06, August 2002.

[40] Jeong J., Gore J., and Peterson B., Mutual information analysis of the EEG in patients with Alzheimer's disease., Clinical Neurophysiology, Vol. 112, No. 5, pp. 827-835, May 2001.

[41] Lempel A. and Ziv J., On the complexity of finite sequences, IEEE Transactions on Information Theory Vol. 22, pp.75-81, 1976.

[42] Ziv J. and Lempel A., A universal algorithm for sequential data compression. IEEE Transactions on Information Theory, Vol. 23, pp. 337-343, 1987.

[43] N. N. Kulkarni, V. K. Bairagi, Extracting Salient features for EEG based diagnosis of Alzheimer disease Using Support vector machine classifier, IETE Journal of Research, Vol. 63, No. 1, pp. 11-22, January 2017. 